\title{
Exact exchange-correlation potential for a time-dependent two-electron system
}

\author{
Irene D'Amico and Giovanni Vignale \\ Department of Physics, University of Missouri, Columbia, Missouri 65211
}

(Received 9 September 1998)

\begin{abstract}
We obtain a solution of the time-dependent Schrödinger equation for a two-electron system confined to a plane by an isotropic parabolic potential whose curvature is periodically modulated in time. From this solution we compute the "exact'" time-dependent exchange correlation potential $v_{x c}$, which enters the Kohn-Sham equation of time-dependent density functional theory. Our " $e x a c t$ " result provides a benchmark against which various approximate forms for $v_{x c}$ can be compared. Finally, $v_{x c}$ is separated in an adiabatic and a pure dynamical part and it is shown that, for the particular system studied, the dynamical part is negligible.
\end{abstract}

[S0163-1829(99)01011-5]

\section{INTRODUCTION}

The time-dependent density-functional theory ${ }^{1-3}$ (TDFT) maps an interacting time-dependent $N$-electron system, described by a Hamiltonian of the form

$$
H=\sum_{i} \frac{p_{i}^{2}}{2 m}+\sum_{i<j} V\left(\mathbf{r}_{i}-\mathbf{r}_{j}\right)+\sum_{i} v_{e x t}\left(\mathbf{r}_{i}, t\right),
$$

with $\mathbf{p}_{i}=-i \hbar \nabla_{i}$ the momentum operator of the single particle, $V\left(\mathbf{r}_{i}-\mathbf{r}_{j}\right)$ the two-particle interaction potential $\left[V\left(\mathbf{r}_{i}\right.\right.$ $\left.-\mathbf{r}_{j}\right)=e^{2} /\left|\mathbf{r}_{i}-\mathbf{r}_{j}\right|$ for Coulomb interaction] and $v_{\text {ext }}\left(\mathbf{r}_{i}, t\right)$ the time-dependent external potential, to a noninteracting time-dependent $N$-electron system having the same density $\rho(\mathbf{r}, t)$. In this formalism the new Hamiltonian, also known as the "Kohn-Sham', (KS) Hamiltonian, can be written as:

$$
H_{K S}=\sum_{i} h_{K S}\left(\mathbf{r}_{i}, \mathbf{p}_{i}, t\right)
$$

where

$$
\begin{aligned}
h_{K S}\left(\mathbf{r}_{i}, \mathbf{p}_{i}, t\right)= & \frac{p_{i}^{2}}{2 m}+v_{e x t}\left(\mathbf{r}_{i}, t\right)+v_{H}\left(\mathbf{r}_{i}, t\right) \\
& +v_{x c}\left([\rho(\mathbf{r}, t)] ; \mathbf{r}_{i}, t\right)
\end{aligned}
$$

is the effective one-particle Hamiltonian. Apart from the external $\left[v_{\text {ext }}\left(\mathbf{r}_{i}, t\right)\right]$ and the Hartree $\left[v_{H}\left(\mathbf{r}_{i}, t\right)\right.$ $\left.=\int d \mathbf{r}^{\prime} \rho\left(\mathbf{r}^{\prime}, t\right) /\left|\mathbf{r}_{i}-\mathbf{r}^{\prime}\right|\right]$ part, the potential contains an "exchange-correlation" $(\mathrm{xc})$ term $\left[v_{x c}\left([\rho(\mathbf{r}, t)] ; \mathbf{r}_{i}, t\right)\right]$ that is an unknown functional of the density. In the TDFT formalism the wave function of the effective noninteracting system is a Slater determinant of $N$ one-particle orbitals $\varphi_{i}(\mathbf{r}, t)$, which satisfies the equation:

$$
h_{K S}(\mathbf{r}, \mathbf{p}, t) \varphi_{i}(\mathbf{r}, t)=i \hbar \frac{\partial}{\partial t} \varphi_{i}(\mathbf{r}, t)
$$

The particle density can then be written as:

$$
\rho(\mathbf{r}, t)=\sum_{i}\left|\varphi_{i}(\mathbf{r}, t)\right|^{2}
$$

As in the time-independent DFT, the main problem in TDFT is to find a good approximation for $v_{x c}([\rho(\mathbf{r}, t)] ; \mathbf{r}, t)$. Among the most used approximations we mention the adiabatic local-density approximation (ALDA), ${ }^{4}$ which is a direct extension of the static LDA to the time-dependent problem, and the optimized effective potential approximation ${ }^{5}$ (OEP) in which $v_{x c}([\rho(\mathbf{r}, t)] ; \mathbf{r}, t)$ is written as a functional of the single-particle orbitals and (usually) only the exchange part is considered. Both approximations determine $v_{x c}([\rho(\mathbf{r}, t)] ; \mathbf{r}, t)$ at time $t$ as a function of the density (or single-particle orbitals) at the same time. Attempts to include the "memory" of the xc potential, i.e., its dependence on the density at earlier times, have been hampered by the fact that such a retarded potential is a severely nonlocal functional of the density, i.e., it does not possess a gradient expansion in terms of the density. ${ }^{6,7}$ For example an early attempt by Gross and $\mathrm{Kohn}^{8}(\mathrm{GK})$ to incorporate retardation within the frame of the LDA was found to be plagued by inconsistencies, such as the failure to satisfy the "harmonic potential theorem,"9 and other exact symmetries. 6,7 Only very recently, ${ }^{6,7,10}$ a consistent local approximation including retardation has been formulated within the frame of the current-density functional theory (CDFT), in which the current density, rather than the density, is used as the basic variable.

In practice, it is not always easy to decide which of the above approximations works best in a concrete application. A comparative study of the performance of different approximations in a simple and well-controlled situation would be very useful. As a first step in this direction, we present, in this paper, an "exact'" (in the sense of highly numerically accurate) calculation of the xc potential for what is probably the simplest nontrivial model of interacting electrons in a time-dependent external potential. This model consists of two electrons, in two dimensions, subjected to a parabolic potential, whose curvature (which is always positive) is periodically modulated in time. A concrete realization of the model could be two electrons in a quantum dot ${ }^{11,12}$ with a time-dependent parabolic confinement potential. We shall show that (i) the time-dependent Schrödinger equation for this system is exactly solvable by a combination of numerical and analytical methods and (ii) the knowledge of the exact solution can be used to compute the exact xc po- 
tential. Our solution for $v_{x c}([\rho(\mathbf{r}, t)] ; \mathbf{r}, t)$ turns out to be the time-dependent generalization of similar calculations recently performed in the static case. ${ }^{13}$ The value of these results lies in the fact that they provide a rigorous benchmark, against which the merits or demerits of various approximate theories can be assessed.

This paper is organized as follows. In Sec. II we discuss the model and the "exact" solution of the corresponding time-dependent Schrödinger equation. In Sec. III we construct the exact xc potential and field $\mathbf{E}_{x c}([\rho(\mathbf{r}, t)] ; \mathbf{r}, t) \equiv$ $-\nabla v_{x c}([\rho(\mathbf{r}, t)] ; \mathbf{r}, t)$ both in the TDFT and in the timedependent CDFT, and we discuss the difference between the two forms. We also compare our result with the known static limit. ${ }^{13}$ In Sec. IV, we draw a comparison between our " $e x$ $a c t$ ' results and the ALDA, OEP, and GK approximations as well as the new approximation presented by Vignale, Ullrich, and Conti ${ }^{10}$ (VUC). In Sec. V we introduce a separation between the adiabatic and the truly dynamic part of $\mathbf{E}_{x c}$. We conclude with a discussion and summary in Sec. VI.

\section{THE MODEL}

We consider two interacting electrons of effective mass $m^{*}$ in a two-dimensional (2D) harmonic potential with frequency $\omega(t)$ periodic in time. The background dielectric constant is $\epsilon$. The corresponding time-dependent Schrödinger equation in atomic units $\left(\hbar=e / \sqrt{\epsilon}=m^{*}=1\right)$ is:

$$
\begin{aligned}
& {\left[-\frac{1}{2}\left(\nabla_{1}^{2}+\nabla_{2}^{2}\right)+\frac{1}{2} \omega^{2}(t)\left(r_{1}^{2}+r_{2}^{2}\right)+\frac{1}{r_{12}}\right] \Psi\left(\mathbf{r}_{1}, \mathbf{r}_{2}\right)} \\
& \quad=i \frac{\partial}{\partial t} \Psi\left(\mathbf{r}_{1}, \mathbf{r}_{2}\right),
\end{aligned}
$$

where $\mathbf{r}_{\mathbf{1}}$ and $\mathbf{r}_{\mathbf{2}}$ are the electronic coordinates and $r_{12}$ is the distance between the electrons. Introducing the center of mass $(\mathrm{CM})$ and relative motion $(\mathrm{RM})$ coordinates $\mathcal{R}=\left(\mathbf{r}_{\mathbf{1}}\right.$ $\left.+\mathbf{r}_{2}\right) / 2$ and $\mathbf{r}=\mathbf{r}_{1}-\mathbf{r}_{2}$, Eq. (6) decouples in the two equations:

$$
\begin{aligned}
& \left(-\frac{1}{4} \nabla_{\mathcal{R}}^{2}+\omega^{2}(t) \mathcal{R}^{2}\right) \Psi_{C M}(\mathcal{R}, t)=i \frac{\partial}{\partial t} \Psi_{C M}(\mathcal{R}, t) \\
& \left(-\nabla_{\mathbf{r}}^{2}+\frac{1}{4} \omega^{2}(t) r^{2}+\frac{1}{r}\right) \Psi_{R M}(\mathbf{r}, t)=i \frac{\partial}{\partial t} \Psi_{R M}(\mathbf{r}, t),
\end{aligned}
$$

where

$$
\Psi\left(\mathbf{r}_{1}, \mathbf{r}_{2}\right)=\Psi_{C M}(\mathcal{R}, t) \Psi_{R M}(\mathbf{r}, t)
$$

is the orbital part of the wave function. The spin state can be either a singlet or a triplet (we assume 3D isotropy for the spin $S$ ). The RM wave function must be even or odd under inversion $\mathbf{r} \rightarrow-\mathbf{r}$, depending on whether $S=0$ or $S=1$, respectively.

For simplicity of notation, we are using ' $\mathbf{r}$ ', to indicate the RM coordinate going back to " $\mathbf{r}_{12}$ "' only where needed to avoid confusion.

\section{A. Solution in the CM channel \\ 1. General analytical solution}

The problem of a quantum harmonic oscillator with a time-dependent frequency has been studied by several authors. ${ }^{14}$ Equation (7) is analytically solvable for a general (periodic or not) $\omega(t)$. The angular momentum is a constant of motion and this allows the separation of angular and radial coordinates. So we obtain the radial equation:

$$
\begin{aligned}
& \left(-\frac{1}{4} \frac{\partial^{2}}{\partial \mathcal{R}^{2}}-\frac{1}{4} \frac{1}{\mathcal{R}} \frac{\partial}{\partial \mathcal{R}}+\omega^{2}(t) \mathcal{R}^{2}+\frac{1}{4} \frac{m^{2}}{\mathcal{R}^{2}}\right) \chi_{n, m}(\mathcal{R}, t) \\
& \quad=i \frac{\partial}{\partial t} \chi_{n, m}(\mathcal{R}, t)
\end{aligned}
$$

where

$$
\Psi_{C M}(\mathcal{R}, t)=\chi_{n, m}(\mathcal{R}, t) \Theta_{m}(\vartheta)
$$

with

$$
\Theta_{m}(\vartheta)=\frac{1}{\sqrt{2 \pi}} e^{-i m \vartheta},
$$

where $m$ is a positive integer denoting the (constant) angular momentum and $\vartheta$ is the angular coordinate of the center of mass.

The general solution of Eq. (10) is given by:

$$
\begin{aligned}
\chi_{n, m}(\mathcal{R}, t)= & \sqrt{\frac{n !}{2^{m}(n+m) !}}\left(\frac{d \phi}{d t}\right)^{(m+1) / 2} \exp \{i(2 n+m+1) \\
& \times[\phi(0)-\phi(t)]\} 2^{m} \mathcal{R}^{m} \\
& \times \exp \left[\left(-\frac{d \phi}{d t}+i \frac{d \ln |X|}{d t}\right) \mathcal{R}^{2}\right] L_{n}^{m}\left(\frac{d \phi}{d t} 2 \mathcal{R}^{2}\right),
\end{aligned}
$$

where $X(t)$ is a complex solution of the classical equation of motion

$$
\begin{gathered}
\ddot{X}(t)=-\omega^{2}(t) X(t), \\
X(t)=|X(t)| e^{i \phi(t)},
\end{gathered}
$$

with a phase $\phi(t)$ satisfying the condition

$$
\frac{d \phi}{d t}>0
$$

The details of the derivation of Eq. (13) are given in Appen$\operatorname{dix} \mathrm{A}$, where it is also shown that such a solution can always be constructed starting from two linearly independent real solutions of Eq. (14).

We stress that Eqs. (12) and (13) provide a complete set of solutions of Eq. (7) for whatever $\omega(t)$, provided that the condition (16) is satisfied.

In the special case of an initial value problem, i.e., if the wave function is specified at $t=0$ as

$$
\Psi_{C M}(\mathcal{R}, 0)=\sum_{n, m} c_{n, m} \chi_{n, m}(\mathcal{R}, 0) \Theta_{m}(\vartheta)
$$


with $\chi_{n, m}(\mathcal{R}, 0) \Theta_{m}(\vartheta)$ the eigenfunctions of $H(0)$, the subsequent time evolution is given by

$$
\Psi_{C M}(\mathcal{R}, t)=\sum_{n, m} c_{n, m} \chi_{n, m}(\mathcal{R}, t) \Theta_{m}(\vartheta),
$$

with the initial condition for $X(t)$

$$
\begin{aligned}
& X(0)=\frac{1}{\sqrt{\omega(0)}}, \\
& \dot{X}(0)=i \sqrt{\omega(0)},
\end{aligned}
$$

where $\omega(0)$ is the frequency of the harmonic oscillator at the initial time.

\section{Floquet ansatz}

If the Hamiltonian is periodic in time (in this case if $\omega(t+T)=\omega(t)$, where $T$ is the period), we can look for a basis set of solutions satisfying the Floquet ansatz: ${ }^{15-17}$

$$
\Psi(t+T)=e^{-i \varepsilon T} \Psi(t),
$$

where $\varepsilon$ (real for bound states) are called Quasi-energies (QE). The $\mathrm{QE}$ are defined modulo $\Omega=2 \pi / T$. This particular basis set has properties that are similar to those of the eigenstates of a static Hamiltonian. ${ }^{15}$

In our calculations, we have chosen for $\omega^{2}(t)$ the form:

$$
\omega^{2}(t)=\omega_{0}^{2}[1+\lambda \cos (\Omega t)] .
$$

To construct Floquet-type solutions of Eq. (10) let us first of all define the Floquet solutions of the classical equation of motion (14) (Refs. 18 and 19) as the solutions $X_{F}(t)$ having the property

$$
X_{F}(t+T)=e^{i K} X_{F}(t) .
$$

There exist two solutions of this kind ${ }^{18,19}$ corresponding to two eigenvalues $e^{i K_{1,2}}$ (with $K_{1}=-K_{2}$ ) either complex conjugate and lying on the unit circle of the complex plane or real and inverse to each other. In the former case the solutions $X_{F}(t)$ remain bounded in time; in the latter, one of them increases exponentially for $t \rightarrow \infty$, a phenomenon known as parametric resonance. The actual value of $K$ as a function of $\lambda$ and $\Omega$ can be calculated from Eq. (14). In this way the $\lambda, \Omega$ plane can be separated in classically stable and unstable regions. The border of the regions of parametric resonance are then defined by the condition: $e^{i K}= \pm 1$.

It is evident, from the form of the general solution (13), that Floquet solutions with real $\mathrm{QE}$ exist within the regions of classical stability. In these regions the two classical Floquet solutions are complex conjugate. This means that one of the two will always satisfy the condition (16) (see also the equivalent condition in Appendix A). If we choose this particular solution as the one that determines the time dependence of $\chi_{n, m}(\mathcal{R}, t)$ in Eq. (13), then the $\left\{\chi_{n, m}(\mathcal{R}, t)\right\}$ form a basis of Floquet wave functions with QE:

$$
\varepsilon_{n, m}=\frac{K}{T}(2 n+m+1) .
$$

To derive Eq. (24) we made use of the relation:

$$
i K=\int_{0}^{T} \frac{\dot{X}}{X} d t=\int_{0}^{T} \frac{d}{d t} \ln |X| d t+i \int_{0}^{T} \frac{d \phi}{d t} d t=i[\phi(T)-\phi(0)] .
$$

The last equality holds because $\int_{0}^{T} d \ln |X| / d t d t=0$ since $\ln |X(t)|$ is periodic. At the border of the classical regions of instability $e^{i K}= \pm 1$, the Floquet solutions $\chi_{n, m}(R, t) \rightarrow 0$ and the set of $\mathrm{QE}\left\{\varepsilon_{n, m}\right\}$ degenerates to $\{0\}$ if $e^{i K}=1$ or to $\{0, \pi / T\}$ if $e^{i K}=-1$.

In the remaining regions of classical instability solutions of the Floquet type cannot be constructed. These regions are centered around the values $\Omega=2 \omega_{0} / k, \quad k=0,1,2 \ldots$ (with $\omega_{0}$ the frequency of the unperturbed harmonic oscillator), ${ }^{18,19}$ so that the value $\Omega=0$ is an accumulation point for the sequence. This means that if $\lambda>0$, it is not possible to perform the limit $\Omega \rightarrow 0$ without entering regions of parametric resonance and so it is not possible to follow the evolution of a Floquet state from a finite $\Omega$ down to 0 . We remark that the occurrence of classical parametric resonance is related to a failure of the conventional Floquet theorem, which ensures the existence of a complete set of Floquet states. The reason is that our harmonic oscillator potential, being not bounded, gives rise to a strictly hermitian Hamiltonian and allows only Floquet states with real QE: evidently, such quasiperiodic states cannot describe the motion of an electron to larger and larger distance from the center that the resonance process would imply. Realistic bounded potentials avoid this problem by allowing the possibility of complex QE in which the electron can escape to infinity (ionization).

\section{B. Solution in the RM channel}

As we did for the CM channel, we separate the angular and radial coordinates in Eq. (8) and we obtain the radial equation:

$$
\begin{aligned}
& \left(-\frac{\partial^{2}}{\partial r^{2}}-\frac{1}{r} \frac{\partial}{\partial r}+\frac{1}{4} \omega^{2}(t) r^{2}+\frac{1}{r}+\frac{l^{2}}{r^{2}}\right) \psi_{n, l}(r, t) \\
& =i \frac{\partial}{\partial t} \psi_{n, l}(r, t)
\end{aligned}
$$

where

$$
\Psi_{R M}(\mathbf{r}, t)=\psi_{n, l}(r, t) \Theta_{l}(\vartheta),
$$

with

$$
\Theta_{l}(\vartheta)=\frac{1}{\sqrt{2 \pi}} e^{-i l \vartheta}
$$

and $l$ a positive integer, even for $S=0$ and odd for $S=1$. Here $\vartheta$ is the angular coordinate for the RM channel. Equation (26) cannot be solved analytically except in the following special cases.

(1) Time independent case. The static limit $(\lambda=0)$ has been well analyzed in three-dimension ${ }^{13}$ and for certain values of the frequency $\omega_{0}$ of the unperturbed harmonic oscillator it is possible to have a completely analytical solution also for the 
RM channel (see Ref. 20). Similarly, it is possible to construct an analytical solution in the two-dimensional case (see appendix B).

(2) Weak correlation limit. We define the weak correlation limit as the regime in which the Coulomb interaction is negligible compared to the harmonic confinement potential. This means that

$$
\frac{l}{a} \rightarrow 0
$$

where $l \equiv \sqrt{\hbar / 2 m^{*} \omega_{0}}$ is the confinement length due to the harmonic potential and $a \equiv \hbar^{2} \epsilon / m^{*} e^{2}$ is the effective Bohr radius. In our units $\left(\hbar=e / \sqrt{\epsilon}=m^{*}=1\right)$ this is equivalent to imposing $\omega_{0} \rightarrow \infty$. In this regime the coulombic term becomes negligible and the RM problem becomes analytically solvable (see part A of this section).

(3) Strong correlation limit in the linear response approximation with respect to $\lambda$. In this limit the Coulomb interaction dominates the harmonic confinement potential. This means

$$
\frac{l}{a} \rightarrow \infty
$$

(so in our units $\omega_{0} \rightarrow 0$ ) and the two electrons can be shown to perform small oscillations about the classical equilibrium position determined by the competition between electrostatic repulsion and harmonic confinement. Expanding the potential energy to the second order in the displacement from the classical equilibrium distance $r_{0} \gg l$ and neglecting corrections of order $l / r_{0}$ to the kinetic energy one obtains the effective harmonic Hamiltonian

$$
H_{e f f}(t)=\frac{p_{r}^{2}}{2 \mu}+\frac{\mu}{2} \widetilde{\omega}^{2}(t)\left(r-r_{0}\right)^{2}-\mu E_{e x t, 1}(t)\left(r-r_{0}\right),
$$

where $p_{r}^{2}=-\partial^{2} / \partial r^{2}, \quad \mu=1 / 2$ is the reduced mass, $\tilde{\omega}^{2}(t)$ $=3 \omega_{0}^{2}+\omega_{1}^{2}(t), \quad \omega_{1}^{2}(t)$ is defined as

$$
\omega_{1}^{2}(t) \equiv \omega_{0}^{2} \lambda \cos (\Omega t),
$$

and $E_{\text {ext }, 1}(t)=-\omega_{1}^{2}(t) r_{0}$ can be viewed as an "external force." Apart from time-dependent phase factors (see Appendix $\mathrm{C}$ for these factors and for details of the derivation) the solution, for $n=l=0$, takes the form:

$$
\Psi(r, t)=\frac{1}{\pi^{1 / 4}}\left(\frac{d \tilde{\phi}}{d t}\right)^{1 / 4} e^{-i \mu \dot{x}_{0}(t)\left(r-r_{0}\right)} e^{(i / 2) \mu(\dot{\tilde{X}} / \tilde{X})\left[r-r_{0}+x_{0}(t)\right]^{2}}
$$

with $\widetilde{X}(t)$ the solution of

$$
\ddot{\tilde{X}}=-\tilde{\omega}^{2}(t) \tilde{X}(t)
$$

$\widetilde{\phi}(t)$ its phase such that $d \widetilde{\phi} / d t>0$ and $x_{0}(t)$ the solution of

$$
\ddot{x}_{0}=-E_{\text {ext }, 1}(t)-\tilde{\omega}^{2}(t) x_{0}(t) .
$$

If we insert in Eq. (33) for $x_{0}(t)$ its linear response approximation expression

$$
x_{0}(t)=-\frac{E_{e x t, 1}(t)}{3 \omega_{0}^{2}-\Omega^{2}}
$$

and for $\widetilde{X}(t)$ the classical Floquet solution of Eq. (34) with $d \widetilde{\phi} / d t>0$, we obtain a Floquet solution for the RM problem.

In the general case Eq. (26) must be solved numerically, and, to construct $v_{x c}(\mathbf{r}, t)$ or $\mathbf{E}_{x c}(\mathbf{r}, t)$, the most natural choice is to consider the dynamical equivalent of the ground state, that is, for the RM channel, the "lowest'" Floquet state $\Psi_{R M}^{0}(r, t)=\psi_{0,0}(r, t) / \sqrt{2 \pi}$. We define this as the state that evolves continuously from the ground state of the static Hamiltonian as the amplitude $\lambda$ of the time-dependent perturbation grows from zero. From now on we only consider $l=0$ (and correspondingly $m=0$ for the CM channel).

In order to calculate this Floquet state we use its property of being an eigenstate of the one-period time-evolution operator $\hat{U}(T)[\Psi(\mathbf{r}, t+T)=\hat{U}(T) \Psi(\mathbf{r}, t)]$ with eigenvalue $e^{-i \varepsilon T}$ [see Eq. (21)]. The idea is to calculate the matrix $\left\{U(T)_{i j}\right\}$ in a suitable basis, diagonalize it and find its "lowest" eigenstate-the "lowest" Floquet state. The basis we choose to calculate $\left\{U(T)_{i j}\right\}$ is the set of eigenstates of a two-dimensional harmonic oscillator with angular momentum equal to zero $\left\{R_{i}(r, 0)\right\}$. For a general instant $t, \quad\left\{U(t)_{i j}\right\}$ are defined by the equation:

$$
R_{j}(r, t)=\sum_{i=1}^{M} U(t)_{i j} R_{i}(r, 0),
$$

where the sum has been truncated for practical purposes. In our calculation $M=60$ - a value that ensures a very good convergence of the lowest QE's. Inserting for each $R_{j}(r, t)$ the expression (37) into Eq. (26), we find for $U(t)_{i j}$ a system of $M$ first-order differential equations. Integrating this system over one period, for each $R_{j}(r, t)$, we obtain $\left\{U(T)_{i j}\right\}$. Since the QE $\varepsilon_{j}$ are defined modulo $\Omega,{ }^{16}$ it is not possible to establish from the value of the eigenvalues of $U(T)$ the "lowest' one. To identify it we have instead used the property that for $\lambda \rightarrow 0, \quad \varepsilon_{j} \rightarrow \varepsilon_{j}^{0}$, where $\varepsilon_{j}^{0}$ is an eigenvalue of the static $(\lambda=0)$ Hamiltonian. ${ }^{15,17}$ In practice we have followed the evolution of the ground-state energy $\varepsilon_{0}^{0}$ for increasing $\lambda$ 's.

\section{CONSTRUCTION AND CALCULATION OF THE "EXACT" EXCHANGE-CORRELATION POTENTIAL}

\section{A. Construction in TDFT}

If we consider two electrons in a singlet state, the KS equations reduce to a single equation for the doubly occupied orbital $\varphi(\mathbf{r}, t)$

$$
\begin{aligned}
& \left\{-\frac{1}{2} \nabla^{2}+v_{e x t}(\mathbf{r}, t)+v_{H}(\mathbf{r}, t)+v_{x c}([\rho(\mathbf{r}, t)] ; \mathbf{r}, t)\right\} \varphi(\mathbf{r}, t) \\
& \quad=i \frac{\partial}{\partial t} \varphi(\mathbf{r}, t) .
\end{aligned}
$$

The KS orbital can be written as

$$
\varphi(\mathbf{r}, t)=|\varphi(\mathbf{r}, t)| e^{i f(\mathbf{r}, t)},
$$


and its modulus is related to the density by the equation:

$$
\rho(\mathbf{r}, t)=2|\varphi(\mathbf{r}, t)|^{2},
$$

while its phase is related to the KS velocity $\mathbf{v}_{K S}(\mathbf{r}, t)$ by

$$
\nabla f(\mathbf{r}, t) \equiv \mathbf{v}_{K S}(\mathbf{r}, t) .
$$

If we insert expression (39) in Eq. (38) and we impose that $v_{x c}([\rho(\mathbf{r}, t)] ; \mathbf{r}, t)$ is real, we obtain two equations, one from the real part of Eq. (38):

$$
\begin{aligned}
& \frac{1}{4} \nabla^{2} \ln \rho(\mathbf{r}, t)+\frac{1}{8}|\nabla \ln \rho(\mathbf{r}, t)|^{2}-v_{e x t}(\mathbf{r}, t)-v_{H}(\mathbf{r}, t) \\
& -v_{x c}([\rho(\mathbf{r}, t)] ; \mathbf{r}, t)-\frac{1}{2}|\nabla f(\mathbf{r}, t)|^{2}-\frac{\partial}{\partial t} f(\mathbf{r}, t)=0,
\end{aligned}
$$

and the second from its imaginary part

$$
\nabla \cdot \nabla f(\mathbf{r}, t)+\nabla f(\mathbf{r}, t) \cdot \nabla \ln \rho(\mathbf{r}, t)+\frac{\partial}{\partial t} \ln \rho(\mathbf{r}, t)=0 .
$$

Equation (42) can be solved for $v_{x c}(\mathbf{r}, t)$ (for simplicity of notation we have dropped the dependence of the xc potential on the density) and we find the following explicit expression:

$$
\begin{aligned}
v_{x c}(\mathbf{r}, t)= & \frac{1}{4} \nabla^{2} \ln \rho(\mathbf{r}, t)+\frac{1}{8}|\nabla \ln \rho(\mathbf{r}, t)|^{2}-v_{e x t}(\mathbf{r}, t) \\
& -v_{H}(\mathbf{r}, t)-\frac{1}{2}|\nabla f(\mathbf{r}, t)|^{2}-\frac{\partial}{\partial t} f(\mathbf{r}, t) .
\end{aligned}
$$

The corresponding expression for the xc electric field is

$$
\mathbf{E}_{x c}(\mathbf{r}, t) \equiv-\nabla v_{x c}(\mathbf{r}, t)
$$

The last two terms of Eq. (44) are peculiar of the timedependent problem while the first four correspond to the static expression ${ }^{13}$ for $v_{x c}$,

$$
v_{x c}^{\text {static }}(\mathbf{r})=\frac{1}{4} \nabla^{2} \ln \rho(\mathbf{r})+\frac{1}{8}|\nabla \ln \rho(\mathbf{r})|^{2}-v_{e x t}(\mathbf{r})-v_{H}(\mathbf{r}) .
$$

Equation (43) is a first-order partial differential equation for $\nabla f$ and is equivalent to the continuity equation for the noninteracting KS system. It shows that $\mathbf{v}_{K S}(\mathbf{r}, t)=\nabla f(\mathbf{r}, t)$ is in general a nontrivial functional of the density. We stress that $\mathbf{v}_{K S}(\mathbf{r}, t)$ is in general not the same as the exact velocity field; only the longitudinal part of the KS current must coincide with the longitudinal part of the physical current due to the continuity equation.

\section{B. Construction in time-dependent CDFT}

The time-dependent CDFT differs from the TDFT in that not only the density but also the current density calculated from the KS single-particle orbitals is exact.

In order to accomplish this, one introduces an $\mathrm{xc}$ vector potential $\mathbf{A}_{x c}$ in the Kohn-Sham equation. 6,7 The KS Hamiltonian $^{7}$ is now:

$$
H^{K S}(t)=\sum_{i}\left\{\frac{1}{2}\left[\mathbf{p}_{i}+\mathbf{A}_{x c}\left(\mathbf{r}_{\mathbf{i}}, t\right)\right]^{2}+v_{H}\left(\mathbf{r}_{\mathbf{i}}, t\right)+v_{e x t}\left(\mathbf{r}_{\mathbf{i}}, t\right)\right\},
$$

which yields both the correct density and current. In the case of two electrons in a singlet state, we get, for the occupied orbital $\varphi(\mathbf{r}, t)$, the time-dependent Schrödinger equation:

$$
\begin{aligned}
& \left(\left\{\frac{1}{2}\left[\mathbf{p}+\mathbf{A}_{x c}(\mathbf{r}, t)\right]^{2}+v_{H}(\mathbf{r}, t)+v_{e x t}(\mathbf{r}, t)\right\}\right) \varphi(\mathbf{r}, t) \\
& \quad=i \frac{\partial}{\partial t} \varphi(\mathbf{r}, t),
\end{aligned}
$$

and we can now follow the same procedure used in TDFT to find an explicit expression for $\mathbf{E}_{x c}(\mathbf{r}, t)$. We obtain:

$$
\begin{aligned}
\mathbf{E}_{x c}(\mathbf{r}, t)= & -\dot{\mathbf{A}}_{x c}(\mathbf{r}, t) \\
= & -\nabla\left[\frac{1}{4} \nabla^{2} \ln \rho(\mathbf{r}, t)+\frac{1}{8}|\nabla \ln \rho(\mathbf{r}, t)|^{2}\right] \\
& -\mathbf{E}_{\text {ext }}(\mathbf{r}, t)-\mathbf{E}_{H}(\mathbf{r}, t)+\nabla\left(\frac{1}{2} v^{2}\right)+\dot{\mathbf{v}}
\end{aligned}
$$

where $\mathbf{v}(\mathbf{r}, t)$ is the exact velocity of the interacting system, $\mathbf{v}(\mathbf{r}, t)=\nabla f(\mathbf{r}, t)+\mathbf{A}(\mathbf{r}, t), \quad \mathbf{E}_{H}=-\nabla v_{H}$ and $\mathbf{E}_{\text {ext }}=-\nabla v_{\text {ext }}$. The expression for the vector potential $\mathbf{A}_{x c}(\mathbf{r}, t)$ follows immediately from Eq. (50) and from the definition Eq. (49). From the imaginary part of Eq. (48) (or equivalently from the continuity equation) we get the first-order partialdifferential equation

$$
\nabla \cdot \mathbf{v}(\mathbf{r}, t)+\mathbf{v}(\mathbf{r}, t) \cdot \nabla \ln \rho(\mathbf{r}, t)+\frac{\partial}{\partial t} \ln \rho(\mathbf{r}, t)=0
$$

The advantage of this formulation is that it expresses $\mathbf{E}_{x c}(\mathbf{r}, t)\left[\right.$ and $\left.\mathbf{A}_{x c}(\mathbf{r}, t)\right]$ as a function of the physical quantities $\mathbf{v}(\mathbf{r}, t)$ and $\rho(\mathbf{r}, t)$.

\section{Circularly symmetric states}

If the time-dependent state is circularly symmetric, as in the case we are studying, then the current is purely radial, therefore purely longitudinal, and the two expressions (45) and (50) coincide [that is $\left.\mathbf{v}(\mathbf{r}, t) \equiv \mathbf{v}_{K S}(\mathbf{r}, t)\right]$. Thus, in this case, there is no difference between the time-dependent DFT and CDFT. Equation (43) can be easily integrated yielding

$$
\frac{\partial}{\partial r} f(r, t)=-\frac{1}{r \rho(r, t)} \int_{0}^{r} \frac{\partial \rho\left(r^{\prime}, t\right)}{\partial t} r^{\prime} d r^{\prime}
$$

\section{Linear response}

In the limit of small external time-dependent perturbation $[\lambda \rightarrow 0$ in Eq. (22)], we expand all the quantities to first order in $\lambda$, i.e., $v_{\text {ext }}=v_{\text {ext }, 0}+v_{\text {ext }, 1}, v_{H}=v_{H, 0}+v_{H, 1}, v_{x c}=v_{x c, 0}$ $+v_{x c, 1}$, and $\ln \rho=\ln \rho_{0}+\rho_{1} / \rho_{0}$ where the subscripts " 0 ", and " 1 ' indicate, respectively, zero and first order with respect to $\lambda$. Then from Eq. (44) we obtain 
TABLE I. Parameters used in our numerical calculations.

\begin{tabular}{lcccc}
\hline \hline & $\omega_{0}$ & $\Omega$ & $\Omega / \omega_{0}$ & $\lambda$ \\
\hline high corr. & $(25-3 \sqrt{33}) / 328 \approx 0.02$ & 0.1 & $\approx 4.2$ & 0.1 \\
low corr. & 1 & 3.2 & 3.2 & 0.1 \\
\hline \hline
\end{tabular}

$$
v_{x c, 0}(r)=\frac{1}{4} \nabla \ln \rho_{0}+\frac{1}{8}\left|\nabla \ln \rho_{0}\right|^{2}-v_{e x t, 0}(r)-v_{H, 0}(r),
$$

and

$$
\begin{aligned}
v_{x c, 1}(r, t)= & -\frac{\partial}{\partial t} f(r, t)+\frac{1}{4}\left(\nabla^{2} \frac{\rho_{1}}{\rho_{0}}+\nabla \ln \rho_{0} \cdot \nabla \frac{\rho_{1}}{\rho_{0}}\right) \\
& -v_{e x t, 1}(r, t)-v_{H, 1}(r, t),
\end{aligned}
$$

where we have neglected also the terms of order $v^{2}(r, t)$ $=|\nabla f(r, t)|^{2}$. Making use of the definition (45), it is easy to recover from Eqs. (53) and (54) the expressions for $\mathbf{E}_{x c, 0}(r)$ and $\mathbf{E}_{x c, 1}(r, t)$.

\section{E. Calculation of the "exact" $v_{x c}(r, t)$}

We have considered in detail two sets of the parameters $\omega_{0}, \Omega$, and $\lambda$ that appear in Eq. (22), corresponding to high and low correlation. The two sets are given in Table I. The values of $\omega_{0}$ have been chosen such that it is possible to construct analytically the solution of the corresponding static Schrödinger equation (see Appendix B). The values of $\lambda$ and $\Omega$ have been chosen so that the system is in the linear response regime, but well above the regions of parametric resonance for the $\mathrm{CM}$ channel and above the first excitation energy of the system.

The "exact' time-dependent densities are plotted in Figs. 1 and 2. For the weak correlation parameter $\omega_{0}=1$ the density is centered at the origin as we can expect from the exact solution in the weak correlation limit (a Gaussian centered at the origin, see Sec. II). In the case of high correlation $\left(\omega_{0}\right.$ $\approx 0.02$ ), on the other hand, the maximum of the density is at finite distance from $r=0$ in agreement with the form that the RM wave function assumes in the strong correlation limit Eq. (33) (an annulus of average radius $r_{0} / 2$, with $r_{0}$ the classical equilibrium distance of the two electrons): the increased strength of the Coulomb repulsion in respect to the harmonic confinement pushes on average the two electrons far from each other. In these plots the solid line represents the static limit while each of the broken lines corresponds to the time dependent $\rho(r, t)$ at different times.

In the insets of Figs. 1 and 2 we plot the time-dependent velocity $\mathbf{v}(r, t)=\nabla f(r, t)$, that is the other necessary ingredient to calculate $v_{x c}(r, t)$ and $\mathbf{E}_{x c}(r, t)$. As the plots show, the motion is approximately a "breathing" motion: the velocity is zero at the origin while for $r \neq 0$ it increases almost linearly. The asymptotic behavior is linear in $r$ with a correction in $1 / r^{2}$. In Fig. $3\left(\omega_{0}=1\right)$ and Fig. $4\left(\omega_{0} \approx 0.02\right)$ we finally plot the results for the potential $v_{x c}(r, t)$ [with the convention $v_{x c}(r, t) \rightarrow 0$ for $\left.r \rightarrow \infty\right]$ and for the field $\mathbf{E}_{x c}(r, t)$ (in the insets). The solid line represents the static limit while each of the broken lines corresponds to $v_{x c}(r, t)\left[\mathbf{E}_{x c}(r, t)\right]$ at different times. We choose to plot also $\mathbf{E}_{x c}(r, t)$, along with

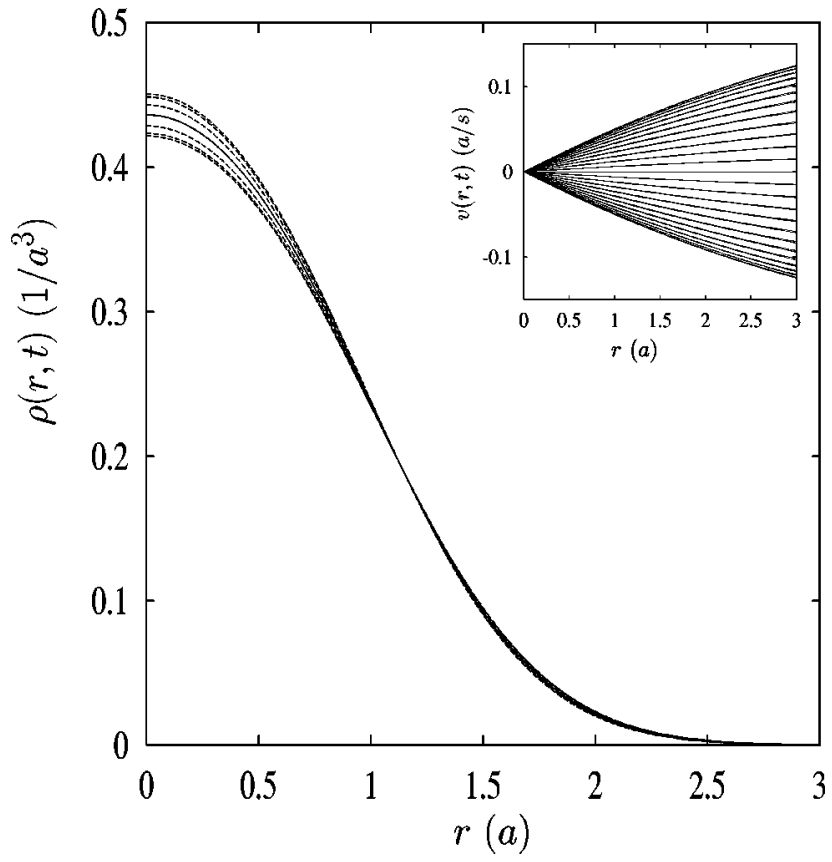

FIG. 1. Lowest Floquet-state electronic density for the weak correlation case $\left(\omega_{0}=1\right)$. The solid line is the exact static result, while each of the broken lines corresponds to different times. In the inset we show the corresponding velocity field $v(r, t)$. Each solid line corresponds to different times.

the more traditional $v_{x c}(r, t)$, since this is the meaningful physical quantity whose asymptotic behavior does not depend on arbitrarily fixed time-dependent constants. As can be seen from the plots, as the correlation in the system increases, the positive peak of $\mathbf{E}_{x c}(r, t)$ for small $r$ increases too. Equivalently the minimum of $v_{x c}$ becomes more pronounced, as the correlation increases, and moves away from the origin. This is related to the enhancement of the strength of the Coulomb repulsion that pushes the maximum of the

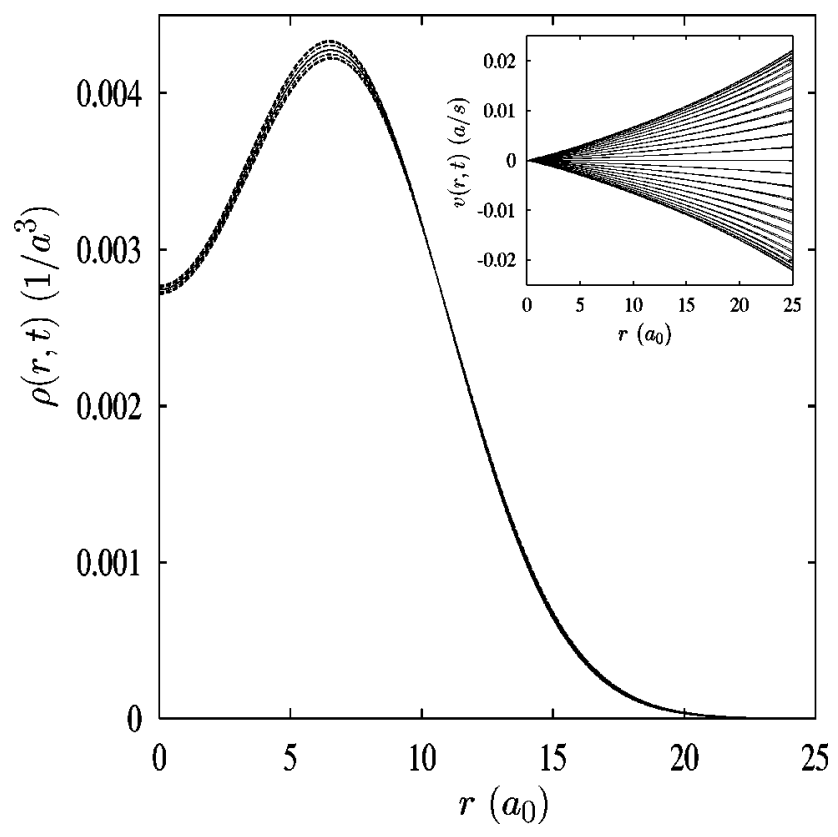

FIG. 2. Same as Fig. 1 but for the strong correlation case $\left(\omega_{0}\right.$ $\approx 0.02$ ). 


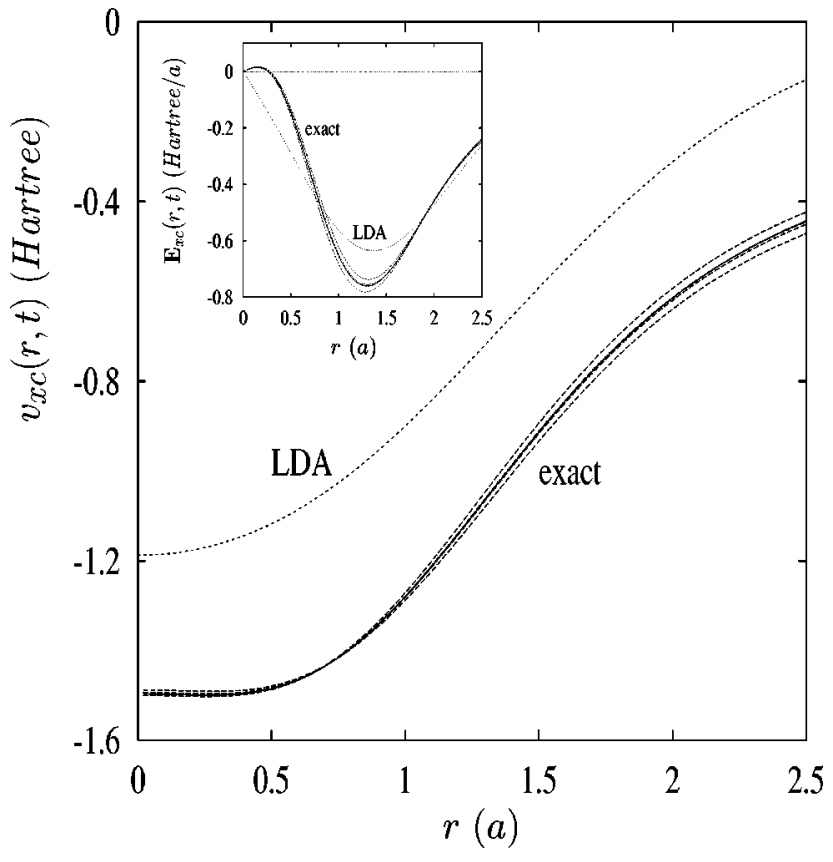

FIG. 3. "Exact'" xc potential $v_{x c}(r, t)$ for the weak correlation case $\left(\omega_{0}=1\right)$. The solid line is the static limit, while each of the broken lines corresponds to $v_{x c}(r, t)$ at different times. Asymptotically $v_{x c}(r, t) \approx-1 / r$, as in the static case. For comparison the static LDA result is also plotted. In the inset we show the corresponding xc field $E_{x c}(r, t)$.

density away from the origin. $\mathbf{E}_{x c}(r, t)$ can be viewed as a force in the KS system which, where positive, contributes to drag the particles away from the origin.

Starting from the asymptotic form of the RM wave function for $r_{12} \rightarrow \infty$, that is, $\Psi_{R M} \propto r_{12}^{\alpha}\left[1+\left(b_{\Re}+i b_{\mathfrak{J}}\right) /\right.$ $r] \exp \left(-r_{12}^{2} \dot{\phi} / 4\right)$, with $\alpha$ real, $b_{\mathfrak{J}}=-\left[\dot{b}_{\mathfrak{R}}+b_{\mathfrak{R}} \ddot{\phi} /(2 \dot{\phi})\right] / \dot{\phi}$

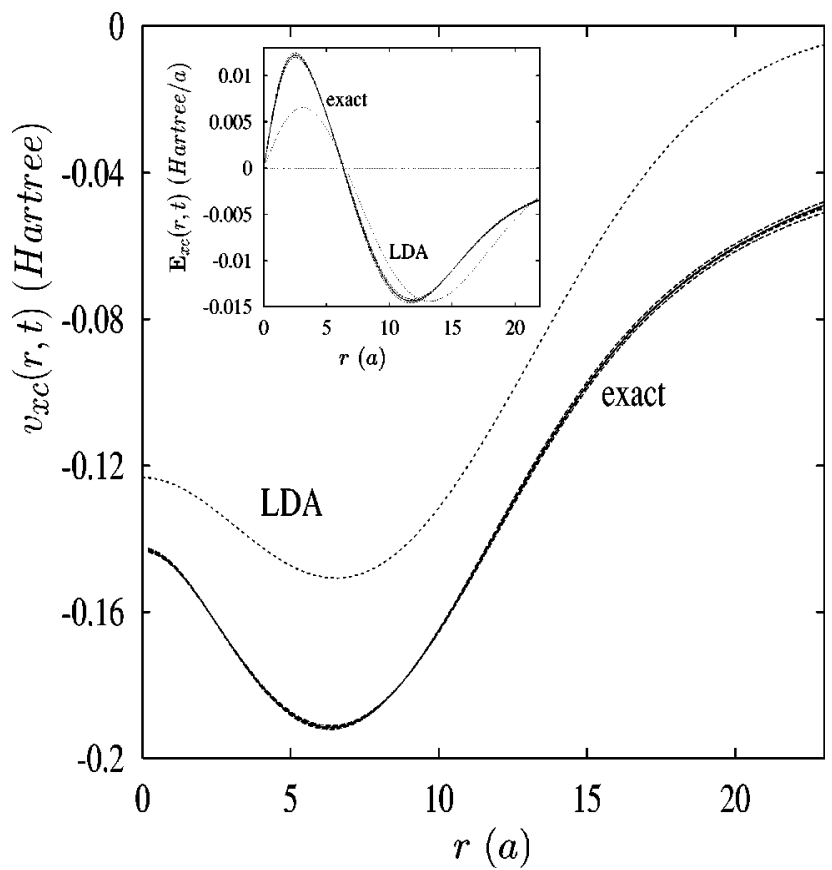

FIG. 4. Same as Fig. 3 but for the strong correlation case $\left(\omega_{0}\right.$ $\approx 0.02$ ). and $b_{\mathfrak{R}}$ defined by $\ddot{b}_{\mathfrak{R}}=-\omega^{2}(t) b_{\mathfrak{R}}+\dot{\phi}$, we get, with a little algebra, the asymptotic form of the density $\left[\rho(r, t) \propto r^{2 \alpha}(1\right.$ $\left.\left.+2 b_{\Re} / r\right) \exp \left(-r^{2} \dot{\phi}\right)\right]$ and of the velocity $[\nabla f(r, t) \approx$ $\left.-r / 2 d(\ln \dot{\phi}) / d t-b_{\mathfrak{J}} / r^{2}\right]$. From these behaviors, using Eq. (44), we can derive the asymptotic behavior for the xc potential that results to be, aside from an irrelevant function of the time only, the same as in the static case: $v_{x c} \approx-1 / r$ for $r \rightarrow \infty$.

In Figs. 3 and 4 we also plot, for comparison, the local density approximation (LDA) of the static $v_{x c}(r)$ and $\mathbf{E}_{x c}(r)$. As can be seen from the figures, in the region plotted, LDA differs from the "exact" result mainly for an irrelevant constant. In fact, if we consider $\mathbf{E}_{x c}$ (insets), LDA behaves, in general, reasonably well except for small $r$ (for which in the weak correlation case has even the wrong sign) and for large $r$ (for which decreases exponentially).

\section{COMPARISON WITH APPROXIMATE THEORIES}

In this section we discuss the comparison between our "exact" result for $v_{x c}(r, t)$ and $\mathbf{E}_{x c}(r, t)$ and the results obtained from the most used approximations, namely the ALDA, the OEP, the GK approximation, and the hydrodynamic approximation recently introduced by VUC. The expressions for the $\mathrm{xc}$ potential in these approximations are as follows.

ALDA: ${ }^{4}$

$$
v_{x c}^{A L D A}(r, t)=\frac{d}{d \rho}\left[\rho(r, t) \varepsilon_{x c}(\rho)\right],
$$

where $\varepsilon_{x c}(\rho)$ is the xc energy per particle of the homogeneous electron gas. In $2 \mathrm{D}$ it is given by

$$
\begin{gathered}
\varepsilon_{x c}=-\frac{4 \sqrt{2}}{3 \pi r_{s}}+\frac{a_{0}}{2} \frac{1+a_{1} \sqrt{r_{s}}}{1+a_{1} \sqrt{r_{s}}+a_{2} r_{s}+a_{3} r_{s}^{3 / 2}} \frac{e^{4} m^{*}}{\hbar^{2} \epsilon}, \\
r_{s}=\frac{1}{\sqrt{\pi \rho}},
\end{gathered}
$$

$a_{0}=-0.3568, \quad a_{1}=1.1300, \quad a_{2}=0.9052, \quad a_{3}=0.4165$

(Refs. 21 and 22)

OEP approximation (which in this simple case is equivalent to the Hartree-Fock approximation) ${ }^{5,2}$

$$
v_{x c}^{O E P}(r, t)=-\frac{1}{2} v_{H}(r, t),
$$

GK $_{\text {approximation }}^{8}$ (valid in the linear response regime):

$$
v_{x c, 1}^{G K}(r, \omega)=\rho_{1}(r, \omega) f_{x c}\left(\rho_{0}, \omega\right),
$$

where $v_{x c, 1}(r, \omega)$ is a Fourier component of $v_{x c, 1}(r, t)$ [defined, with $\rho_{1}(r, t)$, in the "Linear response" section] and $f_{x c, L}\left(\rho_{0}, \omega\right)$ is the longitudinal part of the frequency dependent $x c$ kernel of the homogeneous electron gas.

VUC approximation ${ }^{10}$ for a circularly symmetric potential in $2 \mathrm{D}$ (valid in the linear response regime): 


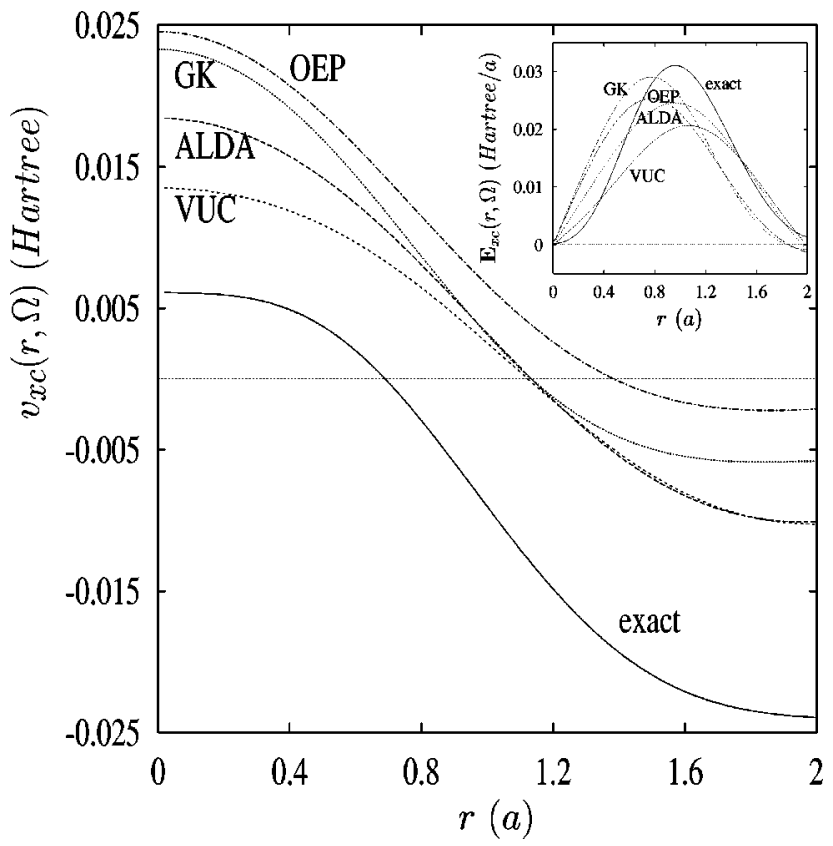

FIG. 5. Comparison between the "exact" first Fourier component $v_{x c}(r, \Omega)$ of the xc potential and some of its most used approximations (weak correlation case $\omega_{0}=1$ ). In the inset we show the corresponding comparison for the xc field $E_{x c}(r, t)$.

$$
\begin{aligned}
v_{x c, 1}^{V U C}(r, \omega)= & v_{x c}^{A L D A}(r, \omega)-\int_{\infty}^{r}\left[\frac { 1 } { \rho _ { 0 } } \left(\nabla \left\{\rho _ { 0 } ^ { 2 } \left[f_{x c, L}\left(\rho_{0}, \omega\right)\right.\right.\right.\right. \\
& \left.\left.-\frac{d^{2} \rho \varepsilon_{x c}}{d \rho^{2}}\right]\left[\frac{v_{1}(r, \omega)}{r}+\frac{\partial v_{1}(r, \omega)}{\partial r}\right]\right\} \\
& \left.\left.-\frac{2}{r} \nabla\left[\rho_{0}^{2} f_{x c, T}\left(\rho_{0}, \omega\right)\right] v_{1}(r, \omega)\right)\right] d r
\end{aligned}
$$

where $v_{1}(r, t)$ is the velocity field and $f_{x c, L}\left(\rho_{0}, \omega\right), f_{x c, T}\left(\rho_{0}, \omega\right)$ are the longitudinal and the transverse part of the frequency dependent $x c$ kernel of the homogeneous electron gas. In Eq. (60) we have made use of the relation $\mathbf{A}_{x c}(r, t)=c \int{ }^{t} \nabla v_{x c}(r, \tau) d \tau$ (see Ref. 10).

In both GK and VUC we have used for $f_{x c}\left(\rho_{0}, \omega\right)$ the expressions recently obtained by Nifosí et al. ${ }^{23}$

The comparison between the "exact" $v_{x c}(r, t)$ and its approximations is made plotting its first Fourier component $v_{x c}(r, \Omega)$. Since our calculations were done in the linear response regime, the difference between $v_{x c}(r, \Omega)$ and $v_{x c, 1}(r, \Omega)$ is negligible. Since the "exact' $v_{x c, 1}(r, \Omega)$ is purely real at nonresonant frequencies, we compare it with the real part of the approximate forms introduced above.

In the case of weak correlation (Fig. 5, $\omega_{0}=1$ ) all the approximations reproduce (apart for an almost constant shift), the general trend. In the strong correlation case (Fig. 6, $\omega_{0} \approx 0.02$ ) the agreement (again apart from a shift) is less good and particularly poor for the OEP. A more detailed analysis can be done considering the xc field $\mathbf{E}_{x c}$ (insets of Figs. 5 and 6), that disregards irrelevant constant shifts. In the case of weak correlation (insets of Fig. $5, \omega_{0}=1$ ) the ALDA reproduces, except for small $r$, the general trend, though underestimating the peak of the potential. In the

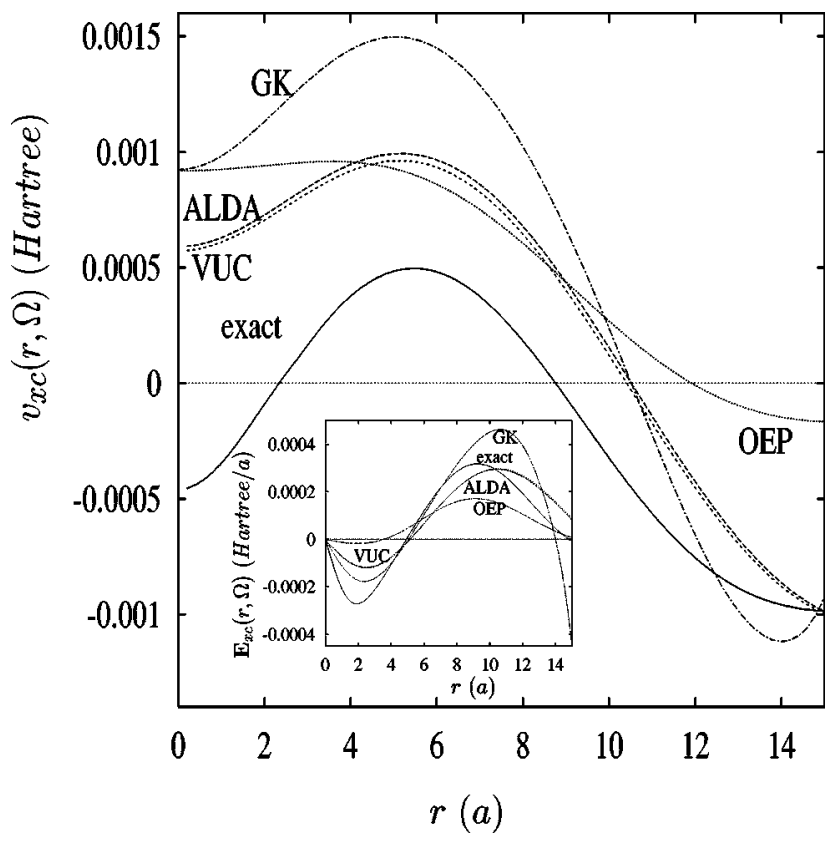

FIG. 6. Same as Fig. 5 but for the strong correlation case $\left(\omega_{0}\right.$ $\approx 0.02$ ).

strong correlation case (inset of Fig. $6, \omega_{0} \approx 0.02$ ) it underestimates the potential for very small $r$, but, for intermediate values, it gets closer to $\mathbf{E}_{x c}(r, \Omega)$. For weak correlation and for small values of $r$ the OEP approximation does not reproduce the "exact" behavior, while in the region in which $\mathbf{E}_{x c}(r, \Omega)$ is significantly nonzero it gets closer to the " $e x$ $a c t$ ' result. In the limit of zero correlation the OEP, which is equivalent to the Hartree-Fock theory for this system, would give the exact result. Its behavior gets worse when the correlation increases (see Fig. 6): it is the only approximation that does not even reproduces the first peak of $\mathbf{E}_{x c}(r, \Omega)$. On the other hand, this is the only approximation that has the correct asymptotic behavior $-1 / r^{2}$ for $r \rightarrow \infty$. In the weak correlation case (Fig. 5) the GK approximation has a behavior similar to the OEP (except for the asymptotic behavior that is not reproduced correctly), while, for strong correlation (Fig. 6) it reproduces the correct trend but underestimates $\mathbf{E}_{x c}(r, \Omega)$ for small values of $r$ and overestimates it for intermediate values. In the case of weak correlation (Fig. 5) the VUC approximation does not reproduce the exact trend for small $r$, while, for intermediate values it get closer to $\mathbf{E}_{x c}(r, \Omega)$ though underestimating its peak. For strong correlation its behavior is almost indistinguishable from the ALDA.

\section{V. "ADIABATIC" AND "DYNAMIC" EXCHANGE CORRELATION POTENTIALS}

Let us focus first on the xc field $\mathbf{E}_{x c}(r, t)$. There has been considerable effort, in recent years, aimed at the construction of a fully dynamic xc potential, which, unlike the ALDA potential, should depend on the density at all previous times, i.e., have a memory. In order to assess the importance of these "memory effects," we shall now separate $\mathbf{E}_{x c}(r, t)$ in an "adiabatic" part $\mathbf{E}_{x c}^{a d}(r, t)$ containing the adiabatic evolution of the static exact $\mathbf{E}_{x c}(r)$ and a "dynamical" one $\mathbf{E}_{x c}^{d y}(r, t)$, peculiar of the time-dependent problem. Compar- 
ing Eqs. (44) and (46), and making use of Eq. (45), it is easy to identify $-\nabla\left[\nabla^{2} \ln \rho(r, t) / 4+|\nabla \ln \rho(r, t)|^{2} / 8\right]-\mathbf{E}_{H}(r, t)$ as adiabatic terms, while $-\nabla\left[-|\nabla f(r, t)|^{2} / 2-\partial f(r, t) / \partial t\right]$ are peculiar to the time-dependent case. However, $\mathbf{E}_{\text {ext }}(r, t)=$ $-\nabla v_{\text {ext }}(r, t)$ is not an explicit functional of the timedependent density and must be treated more carefully. The adiabatic part of $\mathbf{E}_{e x t}(r, t)$ is defined as the electric field $\mathbf{E}_{e x t}^{a d}(r, t)$ which, when applied to the physical interacting system, would yield the exact density $\rho(r, t)$ while the system remains in the instantaneous ground state.

It is then possible to define the "dynamical" part of the external field as what remains after subtracting the adiabatic part:

$$
\mathbf{E}_{e x t}^{d y}(r, t) \equiv \mathbf{E}_{e x t}(r, t)-\mathbf{E}_{e x t}^{a d}(r ; t) .
$$

Now we can separate, in the case of the two electron problem, $\mathbf{E}_{x c}(r, t)$ in an adiabatic $\left[\mathbf{E}_{x c}^{a d}(r, t)\right]$ and dynamical $\left[\mathbf{E}_{x c}^{d y}(r, t)\right]$ part, $\mathbf{E}_{x c}(r, t)=\mathbf{E}_{x c}^{a d}(r, t)+\mathbf{E}_{x c}^{d y}(r, t)$, where

$$
\begin{aligned}
\mathbf{E}_{x c}^{d y}(r, t) & =-\nabla\left\{-\frac{\partial f(r, t)}{\partial t}-\frac{1}{2}[\nabla f(r, t)]^{2}\right\}-\mathbf{E}_{\text {ext }}^{d y}(r, t), \\
\mathbf{E}_{x c}^{a d}(r, t)= & -\nabla\left[\frac{1}{2}\left(\left\{\frac{1}{2} \nabla \ln [\rho(r, t)]\right\}^{2}+\frac{1}{2} \nabla^{2}\{\ln [\rho(r, t)]\}\right)\right] \\
& -\mathbf{E}_{H}(r, t)-\mathbf{E}_{\text {ext }}^{a d}(r, t) .
\end{aligned}
$$

From the definition of $\mathbf{E}_{x c}$, it is straightforward to recover the corresponding expressions for the potential $v_{x c}$.

In the linear regime, using the linearized expression (54) we obtain:

$$
\begin{aligned}
& \mathbf{E}_{x c, 1}^{a d}(r, t)=-\nabla\left(\frac{1}{4} \nabla^{2} \frac{\rho_{1}}{\rho_{0}}+\frac{1}{4} \nabla \ln \rho_{0} \nabla \frac{\rho_{1}}{\rho_{0}}\right)-\mathbf{E}_{e x t, 1}^{a d}(r, t) \\
&-\mathbf{E}_{H, 1}(r, t) \\
& \mathbf{E}_{x c, 1}^{d y}(r, t) \equiv \mathbf{E}_{x c, 1}(r, t)-\mathbf{E}_{x c, 1}^{a d}(r, t) \\
&=\dot{\mathbf{v}}-\mathbf{E}_{e x t, 1}(r, t)+\mathbf{E}_{e x t, 1}^{a d}(r, t)
\end{aligned}
$$

where we have used the fact that $\nabla f(r, t)=\mathbf{v}(r, \mathbf{t})$ and neglected terms of order $v^{2}(r, t)$.

The difficulty in the calculation of $\mathbf{E}_{\text {ext }}^{a d}(r, t)$ is that in general its form is unknown and leads to a nonseparable, two-electron Schrödinger equation. In our case it is possible to calculate analytically $\mathbf{E}_{e x t}^{a d}(r, t)$ and its counterpart $\mathbf{E}_{e x t}^{d y}(r, t)$ in the limit of extremely weak and extremely strong correlation, but for a general set of parameters it will be necessary to find an approximation for $\mathbf{E}_{\text {ext }}^{a d}(r, t)$.

We will now show that $\mathbf{E}_{x c, 1}^{d y}(r, t)$ in this system vanishes exactly in both the weak and strong correlation limits, and it is likely to be very small in the intermediate cases.

\section{A. Calculation of $\mathrm{E}_{x c, 1}^{d y}(r, t)$ \\ 1. "Weak' correlation limit}

In this regime the response of our system to the external potential

$$
v_{\text {ext }}(r, t)=\frac{1}{2}\left[\omega_{0}^{2}+\omega_{1}^{2}(t)\right] r^{2}
$$

with $\omega_{1}^{2}(t)$ defined by Eq. (32), is a "breathing motion," i.e., it can be described as a periodic transformation with a length scale

$$
l(t) \propto\left[\frac{d}{d t} \phi(t)\right]^{-1 / 2} \propto|X(t)| .
$$

Then we can calculate explicitly all the quantities appearing in Eq. (66). The velocity field is given by

$$
\mathbf{v}(r, t)=\frac{\dot{l}(t)}{l(t)} \hat{\mathbf{r}}=\left[\frac{d}{d t} \ln |X(t)|\right] \hat{\mathbf{r}},
$$

$$
\begin{aligned}
\dot{\mathbf{v}}(r, t) & =\left\{\frac{d^{2}}{d t^{2}} \ln [|X(t)|]\right\} \hat{\mathbf{r}} \\
& =\left\{\frac{1}{|X(t)|} \frac{d^{2}}{d t^{2}}|X(t)|-\left[\frac{1}{|X(t)|} \frac{d}{d t}|X(t)|\right]^{2}\right\} \hat{\mathbf{r}},
\end{aligned}
$$

the external field is given by

$$
\mathbf{E}_{e x t, 1}(r, t)=-\omega_{1}^{2}(t) \mathbf{r},
$$

and the "adiabatic", external field is given by

$$
\mathbf{E}_{\text {ext }, 1}^{a d}(r, t)=-\left[\frac{d}{d t} \phi(t)\right]^{2} \mathbf{r} .
$$

This is also the exact $\mathbf{E}_{e x t, 1}^{a d}(r, t)$ corresponding to the noninteracting harmonic oscillator problem.

We can now prove that $\mathbf{E}_{x c, 1}^{d y}(r, t)=0$ in this approximation. Using in Eq. (71) $|X(t)|=X(t) \exp [-i \phi(t)], \quad \ddot{X}=$ $-\omega(t)^{2} X$ and dropping the terms of second order in $\lambda$, we get:

$$
\begin{gathered}
\dot{\mathbf{v}}(r, t)=\left[-\omega_{1}(t)^{2}+\frac{d}{d t} \phi(t)^{2}\right] \mathbf{r} \\
=\mathbf{E}_{\text {ext }, 1}(r, t)-\mathbf{E}_{\text {ext }, 1}^{a d}(r, t)
\end{gathered}
$$

that substituted in Eq. (66) yields $\mathbf{E}_{x c, 1}^{d y}(r, t)=0$.

\section{2. "Strong" correlation limit}

The equilibrium density reduces to a $\delta$-shell $\rho_{0}$ $=2 /\left(\pi r_{0}\right) \delta\left(r-r_{0} / 2\right)$ and we can treat the system classically considering the equation of motion of the separation $r_{12}$ between two classical point charges (see Appendix C). Under the influence of the external potential $v_{\text {ext }, 1}=\omega_{1}(t)^{2} r_{12}^{2} / 2$ the equilibrium separation $r_{12}$ oscillates according to the classical equation of motion

$$
\ddot{r}_{12}=E_{\text {ext }, 1}(t)-3 \omega_{0}^{2}\left(r_{12}-r_{0}\right),
$$

with $E_{\text {ext }, 1}(t)=-\omega_{1}^{2}(t) r_{0}$ and $r_{0}$ is the equilibrium separation in the absence of the external field (we use the linearresponse approximation). We can now define the "adiabatic 
external" field as the one that produces the same deviation from equilibrium as $E_{\text {ext }, 1}(t)$, under static conditions $\left(\ddot{r}_{12}\right.$ $=0)$. That means that

$$
E_{\text {ext }, 1}^{a d}(t)=3 \omega_{0}^{2}\left[r_{12}(t)-r_{0}\right],
$$

where $r_{12}(t)$ is the solution of Eq. (76). From this we can deduce that

$$
\dot{v}_{1}=E_{\text {ext }, 1}(t)-E_{\text {ext }, 1}^{a d}(t),
$$

where $v_{1}$ is the exact velocity field in this limit. This implies that $E_{e x t, 1}^{d y}(t)=0$ in this limit.

\section{Nonextreme cases}

In this case the problem is to find a good approximation for $\mathbf{E}_{\text {ext }}^{a d}(r, t)$. In the case we are considering $\mathbf{E}_{\text {ext }}(r, t)=$ $-\omega(t)^{2} \mathbf{r}$ so in order to have a simple and separable form for $\mathbf{E}_{\text {ext }}^{a d}(r, t)$, we can choose:

$$
\begin{gathered}
\mathbf{E}_{e x t}^{a d}(r, t) \approx-\alpha(t) \mathbf{r}, \\
\mathbf{E}_{e x t}^{d y}(r, t) \approx-\left[\omega(t)^{2} \mathbf{r}-\alpha(t) \mathbf{r}\right],
\end{gathered}
$$

and determine $\alpha$ by optimizing the density.

For the "low-correlation" parameter $\omega_{0}=1$ the approximation $\mathbf{E}_{e x t}^{a d}(r, t)=-(\dot{\phi})^{2} \mathbf{r}$ gives very good results and $\rho_{0}(\mathbf{r} ; t)$ is indistinguishable from $\rho(r, t)$ within the numerical error. The results for the "high-correlation" parameter $\omega_{0}$ $\approx 0.02$ are less good. They can be improved using $\alpha(t)$ $=\omega_{0}^{2}[1+\varepsilon \cos (\Omega t)]$ and tuning the parameter $\varepsilon$. In every case, also in these intermediate cases $\mathbf{E}_{x c}^{d y}(r, t) \approx 0$ within the numerical error.

We conclude that for this particular system the dynamical part of $\mathbf{E}_{x c}(r, t)$ is almost negligible so that the dynamical part of $v_{x c}$ is basically an irrelevant constant. However, we caution that this is at least partly a special feature of the harmonic system studied here (see discussion in the following section) and should not be uncritically generalized to other systems.

\section{DISCUSSION AND SUMMARY}

The comparisons performed in this paper between the " exact" xc potential of a two-electron harmonic atom and several approximate expressions for this quantity, constitute an extremely severe test of the approximations in question. Aside from the exchange-only OEP, all the approximations considered are based on the homogeneous electron gas, and, therefore, are expected to be valid only for systems whose density is slowly varying on the scale of the local average interelectron distance. This condition is certainly not satisfied by our model system-not in the weak correlation regime, in which the length scale of density variation coincides with the average interelectron distance, and much less in the strong correlation regime, in which the latter greatly exceeds the former. In this light, the fact that the ALDA and GK produce xc fields reasonably close to the "exact"' ones, although qualitatively incorrect at large distance from the center, should be regarded as an unexpected success of these approximations.

Another surprising result of our study comes from the separation of $\mathbf{E}_{x c}(r, t)$ into an "adiabatic"' and a purely "dynamical" part. The somewhat counterintuitive result is that, in the case of a time-dependent harmonic external potential, the dynamical part of $\mathbf{E}_{x c}$ is zero in the limits of weak and strong correlation and almost negligible in between. This happens at frequencies well above the first excitation threshold, where the density response is far from adiabatic. In the weak correlation regime, this result depends crucially on the form of the wave function in a parabolic potential. Therefore, we do not expect the conclusion to be generalizable to other potentials. In the strong correlation regime, however, the reduction of the dynamics to harmonic oscillations about a classical equilibrium configuration appears to be a feature of several electronic systems. It is this feature that leads to the vanishing of $\mathbf{E}_{x c}^{d y}(r, t)$ in this regime.

These results throw some light on the surprising ability of the ALDA to give good results even outside its natural domain of validity (low-frequency regime): in a system in which the nonadiabatic corrections are small, a static functional of the density (such as the LDA xc potential), which works well in the static regime, is expected to give a reasonable time-dependent potential upon replacement of the static density with the time-dependent one.

The recently introduced VUC approximation, contains a "dynamical" correction to ALDA [see Eq. (60)] and, in the light of the "exact'" behavior of $\mathbf{E}_{x c}^{d y}(r, t)$ just underlined, it is interesting to notice that the "dynamical" part of VUC is, for this system, small, becoming almost negligible for strong correlation.

In summary, we have found that, for this particular system, the "dynamical" part of $\mathbf{E}_{x c}$ is almost negligible, and the ALDA, GK, and VUC approximations work reasonably well at all coupling strengths (although the VUC underestimates $\mathbf{E}_{x c}(r, t)$ for weak correlation). The OEP, as expected, is reasonable only for weak correlation. The main discrepancies are found to occur at small $r$ and at large $r$ (except for the OEP that has the exact asymptotic behavior). The question of whether these results are generalizable to more complex systems remains open.

\section{ACKNOWLEDGMENTS}

We acknowledge support from NSF Grant No. DMR9706788 and from Research Board Grant No. RB 96-071 from the University of Missouri-Columbia. We thank C. A. Ullrich, S. Conti, R. Nifosí, and C. Filippi for useful discussions.

\section{APPENDIX A}

Making the change of variable $\mathbf{R}=2 \mathcal{R}$, Eq. (7) can be rewritten as

$$
\left(-\nabla_{\mathbf{R}}^{2}+\frac{1}{4} \omega^{2}(t) R^{2}\right) \Psi_{C M}(\mathbf{R}, t)=i \frac{\partial}{\partial t} \Psi_{C M}(\mathbf{R}, t) .
$$

Separating angular and radial coordinates as in Eq. (11), we obtain the radial equation: 


$$
\begin{aligned}
& \left(-\frac{\partial^{2}}{\partial R^{2}}-\frac{1}{R} \frac{\partial}{\partial R}+\frac{1}{4} \omega^{2}(t) R^{2}+\frac{m^{2}}{R^{2}}\right) \chi_{n, m}(R, t) \\
& \quad=i \frac{\partial}{\partial t} \chi_{n, m}(R, t) .
\end{aligned}
$$

Inserting into Eq. (A2) the guess

$$
\chi_{n, m}(R, t)=A(t) R^{m} \exp \left[B(t) R^{2}\right] L_{n}^{m}\left[C(t) R^{2}\right]
$$

(a generalization of the corresponding static solution), we obtain the following equations for the time-dependent coefficients:

$$
\begin{gathered}
i \dot{C}+8 B C+4 C^{2}=0, \\
i \frac{\dot{A}}{A}+4 B+4 m B-4 n C=0 \\
i \dot{B}+4 B^{2}-\frac{1}{4} \omega^{2}(t)=0 .
\end{gathered}
$$

With the ansatz $B=(i / 4)(\dot{X} / X)$, Eq. (A6) becomes

$$
\ddot{X}=-\omega^{2}(t) X,
$$

the classical equation of motion for a harmonic oscillator. The solution $X(t)$ can be written as

$$
X(t)=|X(t)| e^{i \phi(t)}=X_{\mathfrak{R}}+i X_{\mathfrak{I}} .
$$

Since $\chi_{n, m}(R, t)$ must not diverge as $R \rightarrow \infty$, we must impose that the real part of $B(t)=B_{\mathfrak{R}}+i B_{\mathfrak{I}}$ be negative. Using (A8), $B(t)$ can be written as

$$
\begin{aligned}
B(t) & =-\frac{1}{4} \frac{d \phi}{d t}+\frac{i}{4} \frac{d \ln |X|}{d t} \\
& =-\frac{W}{4|X|^{2}}+\frac{i}{4} \frac{d \ln |X|}{d t},
\end{aligned}
$$

where $W=\dot{X}_{\mathfrak{J}} X_{\mathfrak{R}}-\dot{X}_{\mathfrak{R}} X_{\mathfrak{I}}$ is a constant, being the Wronskian of two solutions of Eq. (A7). In order to have a normalizable wave function not identically zero, we have then to impose that $W>0$ or equivalently that $d \phi / d t>0$.

Requiring that $C \in \mathfrak{R}$, from the real part of Eq. (A4) and from Eq. (A10), we get

$$
C(t)=(1 / 2)\left(W /|X|^{2}\right),
$$

which also satisfies the imaginary part of Eq. (A4). Now we can solve Eq. (A5) from which, integrating, we get:

$$
\begin{aligned}
A(t)= & A(0) \cdot \exp \left\{-(m+1) \ln \frac{X(t)}{X(0)}\right. \\
& -i(2 n+m+1)[\phi(t)-\phi(0)]\} .
\end{aligned}
$$

$A(0)$ is determined by the normalization condition $\int_{0}^{\infty}\left|\chi_{n m}(R, t)\right|^{2} R d R=1$,

$$
A(0)=\sqrt{\frac{n !}{2^{m}(n+m) !}\left(\left.\frac{d \phi}{d t}\right|_{t=0}\right)^{(m+1)}} .
$$

Finally, inserting all the expressions for the coefficients in Eq. (A3), after some algebra we get:

$$
\begin{aligned}
\chi_{n, m}(R, t ; X)= & \sqrt{\frac{n !}{2^{m}(n+m) !}}\left(\frac{d \phi}{d t}\right)^{(m+1) / 2} \\
& \times \exp \{i(2 n+m+1)[\phi(0)-\phi(t)]\} R^{m} \\
& \times \exp \left[\left(-\frac{d \phi}{d t}+i \frac{d \ln |X|}{d t}\right) \frac{R^{2}}{4}\right] L_{n}^{m}\left(\frac{d \phi}{d t} \frac{R^{2}}{2}\right) .
\end{aligned}
$$

\section{APPENDIX B}

The solution of static radial equation for the RM channel can be written as:

$$
R_{n, l}(\rho)=\frac{u(\rho)}{\sqrt{\frac{\rho}{\sqrt{\omega_{r}}}}},
$$

with

$$
\begin{gathered}
u(\rho)=e^{-(1 / 2) \rho^{2}} \rho^{s} \sum_{\nu=0}^{\infty} a_{\nu} \rho^{\nu}, \\
\rho \equiv \sqrt{\omega_{r}} r \\
\omega_{r} \equiv \frac{\omega_{0}}{2}, \\
s=\frac{1}{2}+\sqrt{l} .
\end{gathered}
$$

The coefficients of the sum in Eq. (B2) are related by

$$
\begin{gathered}
{\left[s(s+1)-l+\frac{1}{4}\right] a_{1}=\frac{a_{0}}{\sqrt{\omega_{r}}},} \\
{\left[(\nu+s)(\nu+s-1)-l+\frac{1}{4}\right] a_{\nu}-\frac{a_{\nu-1}}{\sqrt{\omega_{r}}}} \\
+\left[\frac{\varepsilon_{r}}{\omega_{r}}-1-2(\nu-2+s)\right] a_{\nu-2}=0,
\end{gathered}
$$

where $\varepsilon_{r}$ is the part of the energy coming from the RM channel and $a_{0}$ is fixed by the normalization condition. Imposing the conditions $a_{n-1} \neq 0, \quad a_{n}=0, \quad a_{n+1}=0$, the sum in Eq. (B2) can be made finite and the coefficients $a_{\nu}$ calculated. From these conditions we also obtain an expression for the energy $\varepsilon_{r}=\omega_{r}(2 n+2 s-1)$ and an expression (less straightforward) for $\omega_{r}$. 
In our calculations we have truncated the sum in Eq. (B2) at $n=2$ obtaining $\omega_{0}=1$ (weak correlation case) and at $n$ $=5$ obtaining $\omega_{0}=(25-3 \sqrt{33}) / 328 \approx 0.02$ (strong correlation case).

\section{APPENDIX C}

In the limit of strong correlation [Eq. (30)] and linearresponse regime the potential energy can be expanded up to the second order about the classical solution and we can also approximate the momentum $\mathbf{p}$ with the radial component $\mathbf{p}_{r}$ $\equiv-i(\partial / \partial r) \hat{r}$ since, in this limit, the dynamic of the problem is basically confined in the $\hat{r}$ direction. The Hamiltonian of the relative motion problem in Eq. (8) can then be approximated as:

$$
H_{e f f}(t)=\frac{p_{r}^{2}}{2 \mu}+\frac{\mu}{2} \tilde{\omega}^{2}\left(r-r_{0}\right)^{2}-\mu E_{e x t, 1}(t)\left(r-r_{0}\right),
$$

where $\mu=1 / 2$ is the reduced mass, $r_{0}=\left(1 / \mu \omega_{0}^{2}\right)^{1 / 3}$ is the classical separation between electrons in the linear regime, $\tilde{\omega}^{2}(t)=3 \omega_{0}^{2}+\omega_{1}^{2}(t)$ and $E_{\text {ext }, 1}(t)=-\omega_{1}^{2}(t) r_{0}$ can be viewed as an "external force." If we define $r_{1}=r-r_{0}$, the deviation from the classical equilibrium position, we can use the change of variable $r_{1}=y-x_{0}(t)$ so that the Hamiltonian becomes: ${ }^{7}$

$$
H(t)=\frac{p_{r}^{2}}{2 \mu}+\frac{\mu}{2} \tilde{\omega}^{2} y^{2}-\mu \tilde{\omega}^{2} x_{0}(t) y-\mu \ddot{x}_{0}(t) y-\mu E_{e x t, 1}(t) y,
$$

where we have dropped the irrelevant terms depending on the time alone. If we impose that

$$
\ddot{x}_{0}=-\tilde{\omega}^{2} x_{0}-E_{\text {ext }, 1}(t) \text {, }
$$

we get

${ }^{1}$ E. Runge and E. K. U. Gross, Phys. Rev. Lett. 52, 997 (1984).

${ }^{2}$ E. K. U. Gross, J. F. Dobson, and M. Petersilka, in Topics in Current Chemistry, edited by R. F. Nalewajski (Springer, Berlin, 1996), Chap. 2.

${ }^{3}$ G. Vignale and W. Kohn, in Electronic Density Functional Theory, edited by J. Dobson, M. K. Das, and G. Vignale (Plenum Press, New York, 1996).

${ }^{4}$ A. Zangwill and P. Soven, Phys. Rev. Lett. 45, 204 (1980).

${ }^{5}$ C. A. Ullrich, U. J. Gossmann, and E. K. U. Gross, Phys. Rev. Lett. 74, 872 (1995)

${ }^{6}$ G. Vignale and W. Kohn, Phys. Rev. Lett. 77, 2037 (1996).

${ }^{7}$ G. Vignale, Phys. Rev. Lett. 74, 3233 (1995).

${ }^{8}$ E. K. U. Gross and W. Kohn, Phys. Rev. Lett. 55, 2850 (1985).

${ }^{9}$ J. Dobson, Phys. Rev. Lett. 73, 2244 (1994).

${ }^{10}$ G. Vignale, C. A. Ullrich, and S. Conti, Phys. Rev. Lett. 79, 4878 (1997).

${ }^{11}$ R. Ashoori, Nature (London) 379, 413 (1996).

${ }^{12}$ M. Wagner, A. V. Chaplik, and U. Merkt, Phys. Rev. B 51, 13 817 (1995).

${ }^{13}$ C. Filippi, C. J. Umrigar, and M. Taut, J. Chem. Phys. 100, 1290

$$
H(t)=\frac{p_{r}^{2}}{2 \mu}+\frac{\mu}{2} \tilde{\omega}^{2} y^{2},
$$

and the problem reduces to a one-dimensional harmonic oscillator and can be solved exactly in a way similar to the one shown for the two-dimensional harmonic oscillator in Appendix A. The general solution takes the form:

$$
\begin{aligned}
\Psi(y, t)= & \left(\frac{1}{2^{n} n ! \sqrt{\pi}}\right)^{1 / 2}\left(\frac{d \tilde{\phi}}{d t}\right)^{1 / 4} e^{(i / 2)(\dot{\tilde{X}} / \tilde{X}) y^{2}} \\
& \times e^{i(1 / 2+n)[\tilde{\phi}(0)-\tilde{\phi}(t)]} H_{n}\left[\left(\frac{d \widetilde{\phi}}{d t}\right)^{1 / 2} y\right],
\end{aligned}
$$

where $H_{n}(x)$ are the Hermite polynomials and $\widetilde{X}(t)$ is a complex solution of the classical equation of motion

$$
\begin{gathered}
\ddot{\tilde{X}}=-\tilde{\omega}^{2}(t) \tilde{X}, \\
\widetilde{X}(t)=|\widetilde{X}(t)| e^{i \tilde{\phi}(t),}
\end{gathered}
$$

with a phase $\widetilde{\phi}(t)$ satisfying the condition $d \widetilde{\phi} / d t>0$. The solution of the original problem Eq. (C1) with $n=0$ is, therefore,

$$
\begin{aligned}
\Psi(r, t)= & \frac{1}{\pi^{1 / 4}}\left(\frac{d \tilde{\phi}}{d t}\right)^{1 / 4} e^{(i / 2)[\tilde{\phi}(0)-\tilde{\phi}(t)]} e^{-i \mu \dot{x}_{0}(t)\left(r-r_{0}\right)} \\
& \times e^{(i / 2) \mu(\dot{\tilde{X}} / \tilde{X})\left[r-r_{0}+x_{0}(t)\right]^{2}} \\
& \times \exp \left[i \int_{0}^{t} d t^{\prime}\left(\frac{\mu}{2} \tilde{\omega}^{2} x_{0}^{2}-\frac{1}{2} \mu \dot{x}_{0}^{2}\right)\right]
\end{aligned}
$$

We stress that in the regime of high correlation $\Lambda / r_{0} \rightarrow 0$, where $\Lambda \propto \omega_{0}^{1 / 2}$ the width of the Gaussian entering the solution (C8), the wave function is concentrated around $r_{0}$ [that justifies the approximation $(\mathrm{C} 1)]$ and tends to a $\delta$ function in the extreme limit.
(1994); C. Filippi, X. Gonze, and C. J. Umrigar, in Recent Developments and Applications of Density Functional Theory, edited by J. M. Seminario (Elsevier, Amsterdam, 1996).

${ }^{14}$ K. Husimi, Prog. Theor. Phys. 9, 381 (1953); M. Kleber, Phys. Rep. 236, 6 (1994); I. A. Pedrosa, Phys. Rev. A 55, 3219 (1997).

${ }^{15}$ H. Sambe, Phys. Rev. A 7, 2203 (1973).

${ }^{16}$ S. I. Chu, Adv. At. Mol. Phys. 21, 197 (1985), and references therein.

${ }^{17}$ B. Galdrikian, Ph.D. thesis, University of California, Santa Barbara, 1994.

${ }^{18}$ V. I. Arnold, Mathematical Methods of Classical Mechanics (Springer-Verlag, New York, 1978), Sec. 25.

${ }^{19}$ L. D. Landau and E. Lifshitz, Mechanics, in Course of Theoretical Physics, 3rd ed. (Pergamon Press, Oxford, 1976), Vol. 1, Sec. 27.

${ }^{20}$ M. Taut, Phys. Rev. A 48, 3561 (1993).

${ }^{21}$ A. Isihara and T. Toyoda, Z. Phys. B 29, 70 (1978).

${ }^{22}$ B. Tanatar and D. M. Ceperley, Phys. Rev. B 39, 5005 (1989).

${ }^{23}$ R. Nifosí, S. Conti, and M. P. Tosi, Phys. Rev. B 58, 12758 (1998). 